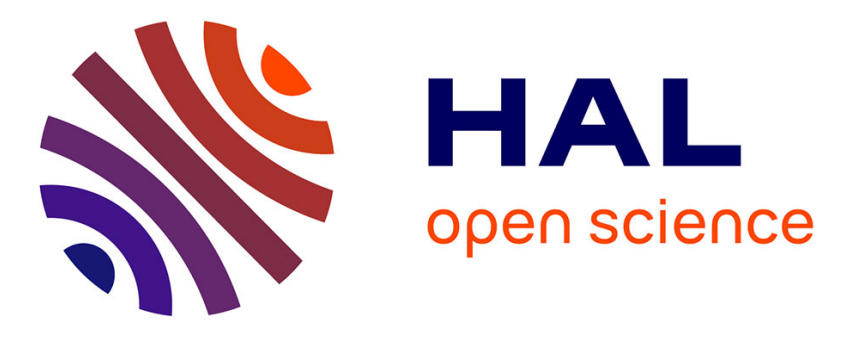

\title{
Influence of afterbody rounding on the pressure distribution over a fastback vehicle
}

Giacomo Rossitto, Christophe Sicot, Valérie Ferrand, Jacques Borée, Fabien Harambat

\section{- To cite this version:}

Giacomo Rossitto, Christophe Sicot, Valérie Ferrand, Jacques Borée, Fabien Harambat. Influence of afterbody rounding on the pressure distribution over a fastback vehicle. Experiments in Fluids, 2016, 57 ( 3), pp.1-12. 10.1007/s00348-016-2120-1 . hal-01297591

\section{HAL Id: hal-01297591 \\ https://hal.science/hal-01297591}

Submitted on 4 Apr 2016

HAL is a multi-disciplinary open access archive for the deposit and dissemination of scientific research documents, whether they are published or not. The documents may come from teaching and research institutions in France or abroad, or from public or private research centers.
L'archive ouverte pluridisciplinaire HAL, est destinée au dépôt et à la diffusion de documents scientifiques de niveau recherche, publiés ou non, émanant des établissements d'enseignement et de recherche français ou étrangers, des laboratoires publics ou privés. 


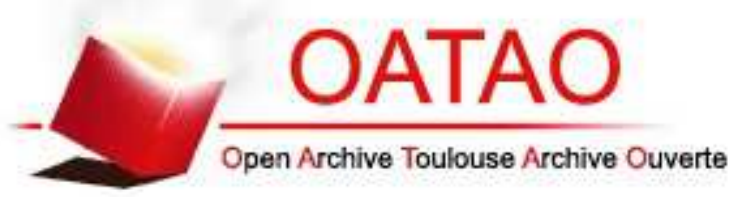

\section{Open Archive TOULOUSE Archive Ouverte (OATAO)}

OATAO is an open access repository that collects the work of Toulouse researchers and makes it freely available over the web where possible.

This is an author-deposited version published in : http://oatao.univ-toulouse.fr/ Eprints ID : 15653

To link to this article : DOI : $10.1007 /$ s00348-016-2120-1

URL : http://dx.doi.org/10.1007/s00348-016-2120-1

To cite this version : Rossitto, Giacomo and Sicot, Christophe and Ferrand, Valérie and Borée, Jacques and Harambat, Fabien Influence of afterbody rounding on the pressure distribution over a fastback vehicle. (2016) Experiments in Fluids, vol. 57 ( $\left.\mathrm{n}^{\circ} 3\right)$. pp. 1-12. ISSN 0723-4864

Any correspondence concerning this service should be sent to the repository administrator: staff-oatao@ listes-diff.inp-toulouse.fr 


\title{
Influence of afterbody rounding on the pressure distribution over a fastback vehicle
}

\author{
Giacomo Rossitto $^{1,2}$ (D) Christophe Sicot $^{2} \cdot$ Valérie Ferrand $^{3} \cdot$ Jacques Borée $^{2}$. \\ Fabien Harambat ${ }^{1}$
}

\begin{abstract}
Experimental analyzes were performed to understand the drag evolution and the flow field modifications resulting from afterbody rounding on the Ahmed body, a simplified vehicle model with 25 degrees rear slant. Curvature effects were investigated using balance measurements, flow visualizations, wall pressure, and particle image velocimetry measurements. The rear end of the original well-known Ahmed body has sharp connections between the roof and the rear window as well as squared rear pillars. Similarly to previous studies, rounding the roof/backlight intersection was shown to reduce drag up to $16 \%$. Surprisingly, additional rear curvature associated with side pillar rounding did not further modify the drag. However, the zero net effect was found to result from opposite drag effects on the slanted and vertical surfaces and to hide strong local modifications on the flow field. The tridimensional organization and vorticity transport in the near wake were analyzed and connected to the observed local increase in the pressure drag on the base. Finally, these results were shown to be generic for a realistic rounded-end car shape.
\end{abstract}

Giacomo Rossitto

giacomo.rossitto@ensma.fr

1 PSA Peugeot Citroën, 2 Route de Gisy, 78943 Velizy-Villacoublay, France

2 Institut Pprime, UPR-3346 CNRS, ENSMA, Université de Poitiers, Poitiers, France

3 Institut Supérieur de l'Aéronautique et de l'Espace (ISAE-SUPAERO), Université de Toulouse, Toulouse, France

\section{Introduction}

Facing strict rules for exhaust gases imposed by the European Community at the 2020 horizon $\left(95 \mathrm{~g} / \mathrm{km}\right.$ of $\left.\mathrm{CO}_{2}\right)$, car manufacturers have to further reduce fuel consumption and $\mathrm{CO}_{2}$ emissions. This purpose can be achieved by improving engine efficiency and by reducing energy losses. In this context, significant effort is being put into drag reduction, by means of shape optimization. Rounding the rear part of the vehicle appears to be an interesting solution, but a compromise has to be found between style and aerodynamic efficiency. Most of the fundamental aerodynamic analyzes have been carried out on simplified car models featuring sharp edges at the rear (Ahmed et al. 1984; Davis 1982; Howell and Hickman 1997). Only recently have very few papers addressed the question of rear edge curvature and aerodynamic performances. Thacker et al. (2012) showed that rounding the edge between the roof and the rear slant on the Ahmed body results in a $10 \%$ drag reduction. Authors attributed this reduction to the fully attached flow over the backlight and the downstream motion of the structures developing in the near wake.

Fuller et al. (2014) analyzed the benefits of rounding the rear pillar geometry on the Davis (1982) model. They observed that rounded edges generated a different wake structure dominated by an interaction between the longitudinal vortices and the separated region, resulting in a drag reduction of $11 \%$.

Based on the literature review, it appears that rounding afterbodies sizably affected aerodynamic loads and flow fields, but systematic investigation into the effects of changing radiuses of upper and side backlight edges was not reported. The present study addresses this question by rounding alternatively and then, simultaneously, the roof and lateral edges of the backlight of an Ahmed model. 
Particular attention was given to understanding how the modified flow on the backlight interacts with the near wake and promotes drag changes.

Three models were used with different afterbody curvatures. To fully characterize the flow pattern on the afterbody and the near wake, wall pressure measurements on the slanted and base surfaces were completed by skin friction line visualizations and stereo-PIV velocity fields.

The description of the experimental setup in Sect. 2 is followed by the comparative analysis of the drag force developing on the three tested configurations. The role of pillar rounding in flow behavior and drag generation is then discussed in Sect. 4. Finally, main conclusions and perspectives of the study are presented. A further study, briefly outlined in "Appendix", generalizes the results obtained through exploring flow features on a more realistic car body.

\section{Experimental setup}

All the tests were conducted in the PSA Peugeot Citroën wind tunnel of La Ferté Vidame (France). This Eiffel wind tunnel is $52 \mathrm{~m}$ long and has a test section which is $2 \mathrm{~m}$ high, $5.2 \mathrm{~m}$ wide, and $6 \mathrm{~m}$ long. The maximum speed is $53 \mathrm{~m} / \mathrm{s}$. The wind tunnel blockage ratio was $1.4 \%$. The model was placed on a elevated surface as high as approximately half the length of the model, Beaudoin and Aider (2008). The nomenclatures of flow and the geometrical features are reported in Fig. 1. The schematic of the model, the relevant dimensions, and the coordinate system are reported in Fig. 2, [see Ahmed et al. (1984) for a complete description of the body]. The reference length, $\xi=201 \mathrm{~mm}$, is the

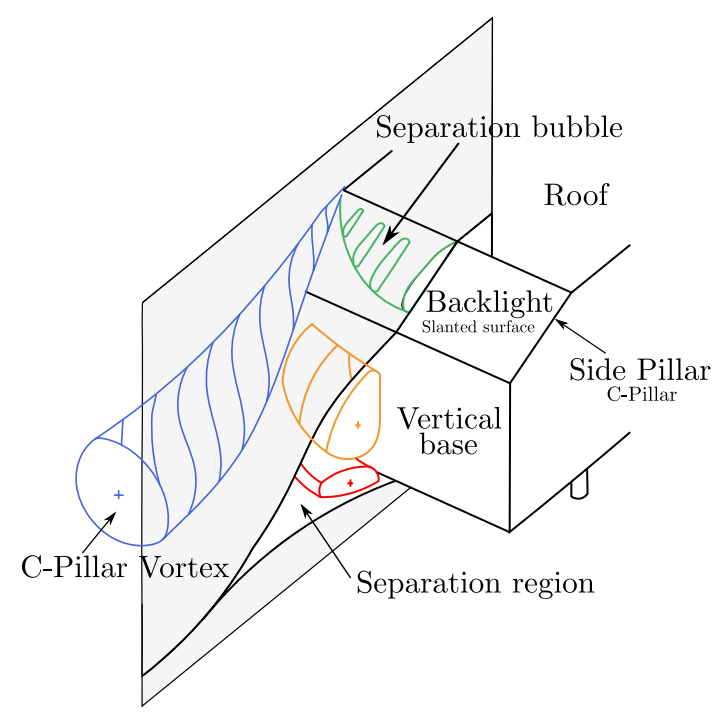

Fig. 1 Nomenclature of flow and geometrical features

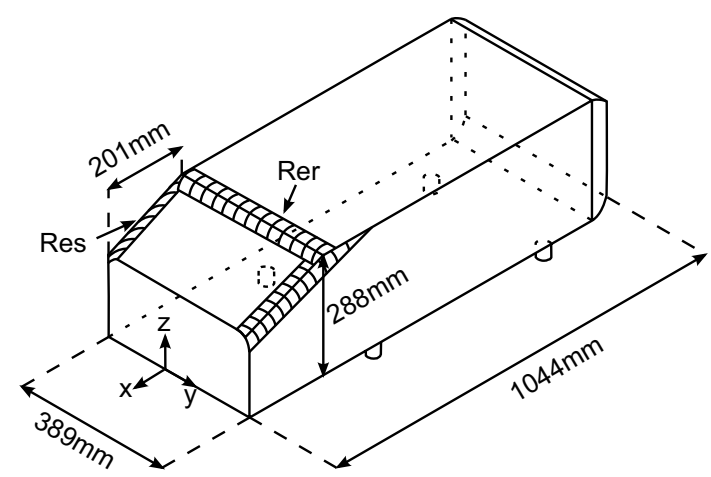

Fig. 2 Ahmed body and location of the rounded edges. Rer stands for rounded edge at the end of the roof and Res for rounded edge on the side

length of the horizontal projection of the model's rear window. Normalized lengths are noted as starred values. The origin of the Cartesian coordinate system is on the symmetry plane. $X^{*} \in[-1,0]$ is the region over the rear slant and $X^{*} \geq 0$ the near wake. The locations of the rounded edges are reported in Fig. 2: Rer stands for the upper edge of the backlight, at the roof junction, and Res for the side edges. The models are then called $R_{\alpha} S_{\beta}$, where $R$ stands for Roof and $S$ for Side. $\alpha$ and $\beta$ represent the values of the radii. The radius of curvature of the afterbody is expressed as a percentage of the reference length. The reference case with sharp edges (i.e. no curvature) is referred as $R_{0} S_{0}$. The second model with a rounded edge of $10 \%$ between the roof and the rear window is referred $R_{10} S_{0}$. Finally, the model with $10 \%$ curvature both on the upper and side edge of the backlight is noted $R_{10} S_{10}$. An initial numerical study was conducted to select the roof curvature radius Rer, Rossitto et al. (2014). It is interesting to notice that drag and lift values obtained with $R_{5} S_{0}$ were very similar to the $R_{40} S_{0}$ ones. Once the backlight separation bubble is suppressed, further increase in roof edge radius has no effect on aerodynamic loads. $10 \%$ was chosen as the reference roof edge radius, and the same pillar radius was selected for the $R_{10} S_{10}$ model. At this Reynolds number, it was shown that this moderate rounding induces strong local modifications of the flow. However, as in Fuller and Passmore (2014), it was found that separation of the flow along the side and over the rounded C-pillars still occurred. For higher ratios of rounding $\left(R_{10} S_{>20}\right.$ according to the preliminary numerical study), the side radius is large enough to prevent side flow separation, this case will be briefly described in the "Appendix" for a more realistic model. $R_{10} S_{10}$ is therefore an intermediate configuration between the sharp side $R_{10} S_{0}$ and high side radius (typically Res $>20 \%$ ). It was chosen to concentrate on this configuration.

The free stream velocity $U_{0}$ was set at $40 \mathrm{~m} / \mathrm{s}$, yielding a Reynolds number based on the length of the model of 2.6 
$10^{6}$. All velocities are normalized with $U_{0}$ and are noted as starred values. To evaluate the aerodynamic coefficients, a six-component balance was used. The drag and lift coefficients were calculated as follows:

$C_{\mathrm{d}, 1}=\frac{F_{\mathrm{d}, 1}}{\frac{1}{2} \rho U_{0}^{2} S}$

where $F$ is the force measured by the balance, $\rho$ is the density of the air, and $S$ is the frontal surface area of the model. The precision of the balance was 0.001 for the drag coefficient $\mathrm{Cd}$, and 0.002 for the lift coefficient $\mathrm{Cl}$. The static wall pressure coefficient at one point $i$ was computed from the expression:

$C_{\mathrm{P}}(i)=\frac{P(i)-P_{0}}{\frac{1}{2} \rho U_{0}^{2}}$

where $P(i)$ is the static pressure of point $(i), P_{0}$ the static pressure measured upstream of the model. The left-hand side of the models was equipped with 115 pressure probes connected to a SCANdaq 8000 acquisition system. The acquisition rate was $40 \mathrm{~Hz}$ for 3000 samples, thus giving 75 s of time recording.

Furthermore, PIV measurements were acquired in the wake of the Ahmed body. The laser sheet was set by a $2 \times 120$ mJ Nd:Yag Quantum Big Sky Laser. The Dantec Flowsense 4M mkII (2024 pixels $\times 2024$ pixels) equipped with 105-mm lenses generated $462 \mathrm{~mm} \times 462 \mathrm{~mm}$ fields of view. 2D PIV was performed on $x z$ planes: $Y^{*}=0$, $Y^{*}=0.49$ and $Y^{*}=0.97$. Twenty-six $z y$ planes were recorded using the stereo-PIV techniques to obtain the three velocity components of the flow field: six planes over the rear window, $X^{*} \in[-1,0]$ with a non-uniform spacing between them and 20 planes in the near wake, $X^{*} \in[0,0.5]$ every $\Delta X^{*}=0.025$. Post-processing was performed with a final interrogation window of $16 \times 16$ pixels, after an initial window of $32 \times 32$ pixels, with an overlap of $50 \%$ in the horizontal and vertical directions. Grid spacing was then $1.82 \mathrm{~mm}$. Eight hundred images were recorded with a $7 \mathrm{~Hz}$ trigger rate. With this setting, the $95 \%$ confidence limit represents approximately $\pm 3 \%$ of the mean velocity.

Stereo-PIV data were also analyzed by using the $\Gamma_{2}$ criterion. This vortex identification algorithm allows the identification of regions of dominant rotation in a flow with an integral and normalized criterion. It was introduced by Graftieaux et al. (2001) in order to identify vortices for unsteady turbulent flows. The $\Gamma_{2}$ criterion, taking into account the average convection velocity in the domain $S$, is a Galilean invariant. $\Gamma_{2}$ is defined by the relation:

$$
\begin{aligned}
\Gamma_{2} & =\frac{1}{S} \iint_{S} \frac{\left[\mathbf{P M} \wedge\left(\mathbf{U}_{\mathbf{M}}-\mathbf{U}_{\mathbf{P}}\right)\right] \cdot \mathbf{e}_{\mathbf{x}}}{\|\mathbf{P M}\| \cdot\left\|\mathbf{U}_{\mathbf{M}}-\mathbf{U}_{\mathbf{P}}\right\|} \mathrm{d} S \\
\mathbf{U}_{\mathbf{P}} & =\frac{1}{S} \iint_{S} \mathbf{U d} S
\end{aligned}
$$

where $S$ is a two-dimensional area surrounding $P$ and $M$ points in $S$. A dominant rotation is then defined for $\frac{2}{\pi} \leq\left|\Gamma_{2}\right| \leq 1$. In what follows, the domain $S$ was chosen to include 24 PIV velocity vectors surrounding $P$.

\section{Global analysis of the drag force}

In this section, the aerodynamic drag and lift of the three tested configurations are presented and analyzed in terms of pressure distribution and global flow structure in the near wake.

\subsection{Aerodynamic coefficients}

Measurements of drag and lift coefficients are summarized in Table 1 for the three tested configurations $R_{0} S_{0}$ (reference case), $R_{10} S_{0}$ and $R_{10} S_{10}$. The analysis is based on the relative variation with respect to the reference case. For the reference case, the measured Cd coefficient is found to be higher than the one presented by Ahmed et al. (1984) $\left(C_{\mathrm{d}}=0.285\right.$ at $60 \mathrm{~m} / \mathrm{s})$; nevertheless, this value is consistent with other studies such as Conan et al. (2011) and Joseph et al. (2012). The recirculation length on the rear window appears to be very sensitive to the quality of the upper sharp edge, Joseph (2012). Differences in bubble lengths could explain deviations on the drag coefficient between similar studies. This is not a hindrance for this work because the purpose is the relative comparison between the three tested models.

When rounding the backlight upper edge $\left(R_{10} S_{0}\right.$ model $)$, a subsequent $16 \%$ drag reduction is observed. With a $40 \%$ roof curvature radius, Thacker et al. (2012) measured a comparable reduction, i.e. $10 \%$. Explanations on the origin of the drag reduction will be developed in the following sections. The addition of rear curvature with rounded pillars does not induce any changes in the overall drag: The $R_{10} S_{10}$ model shows the same drag coefficient as the $R_{10} S_{0}$

Table 1 Aerodynamic coefficients and their variation with respect to the reference case $R_{0} S_{0}$

\begin{tabular}{lllll}
\hline & $\mathrm{Cd}$ & $\mathrm{Cl}$ & $\Delta \mathrm{Cd}$ & $\Delta \mathrm{Cl}$ \\
\hline$R_{0} S_{0}$ & 0.356 & 0.311 & - & - \\
$R_{10} S_{0}$ & 0.297 & 0.333 & $-16 \%$ & $+7 \%$ \\
$R_{10} S_{10}$ & 0.298 & 0.254 & $-16 \%$ & $-18 \%$ \\
\hline
\end{tabular}


one. This point will be shown to result from strong local opposite effects on the slanted and vertical surfaces.

Regarding lift coefficient, the $R_{10} S_{0}$ case shows a moderate increase when compared to the sharp edge configuration. This increase is caused by flow acceleration around the rounded upper edge. It can be noticed that in automotive aerodynamics, increasing lift is detrimental in terms of stability and braking distance, Katz (1995). A strong reduction in lift is observed for the case $R_{10} S_{10}$. It will be hereunder shown that the global evolution of the pressure recovery over the slanted surface and the reduction in the intensity of the longitudinal vortices are responsible for this positive effect.

\subsection{Interpretation of static wall pressure distribution and velocity fields in the plane of symmetry}

\subsection{1 $R_{0} S_{0}$ configuration}

For the reference configuration $R_{0} S_{0}$, Ahmed et al. (1984) identified three main sources of pressure drag generated along the slanted surface and in the near wake of the body. Due to the sharp edge between the roof and the backlight, the flow experiences separation-reattachment, thereby forming a separated bubble along the slanted surface. The length of the recirculation zone was found here to cover $75 \%$ of the rear window, which is consistent with the available literature, Joseph (2012).

The second drag source is associated with the highly energetic longitudinal vortex, the so-called C-pillar vortex, developing along the rear side pillars. Its presence leads to a strong localized low pressure on the side of the rear slant, shown on the right side in Fig. 3.

The third major source of drag is associated with the separated region in the near wake (left side of Fig. 3) leading to the low-pressure distribution along the vertical base (right side of Fig. 3). It is useful to define here a variable $L^{*}$ as the length of the mean recirculation region identified by the saddle point in the streamlines of the near wake. Precisely, $L^{*}=0.89$ for $R_{0} S_{0}$ (see Fig. 3 ). $L^{*}$ will be used in Sect. 4 as a characteristic length for the crossflow analysis.

\subsection{2 $R_{10} S_{0}$ configuration}

When rounding the upper edge of the slanted surface, noticeable changes are observed in the flow field. No separation occurs at the junction between the roof and the rear slant. A low-pressure area around the rounded edge is generated, followed by a pressure recovery over the rear slant (Fig. 4). The flow remains fully attached until it separates at the end of the rear window. On the side of the model, the C-pillar vortex is still present, as illustrated in Fig. 4 by the concentrated streamwise vorticity. The low-pressure footprint of the longitudinal vortex does not exhibit any significant differences compared with the reference case. The time-averaged streamlines in the plane of symmetry of the near wake show a counter-rotating system more symmetrical than the $R_{0} S_{0}$ configuration. Moreover, those structures, related to low static pressure, are shifted further downstream, thus increasing the mean recirculation bubble by $12 \%\left(L^{*}=1\right)$ over the reference sharp case. To help interpreting the origin of lift and drag alteration for the rounded Ahmed bodies, the static pressure coefficient has been integrated separately over the slanted surface and the vertical base. Table 2 reports the percentage change in the pressure coefficient for the $R_{10} S_{0}$ and $R_{10} S_{10}$ models compared to $R_{0} S_{0}$. Although the lack of pressure probes along the rounded pillars makes those results an estimation, it appears clearly that the downstream motion of the wake structures leads to an increase in base pressure of up to $28 \%$ for the $R_{10} S_{0}$ configuration.

\subsection{3 $R_{10} S_{10}$ configuration}

One important observation made during this study, discussed in more detail in Sect. 4 , is that the rounding of the C-pillar displaces the origin of the longitudinal vortex toward the base of the body along the C-pillar. This effect is also described for flows over delta wings featuring blunt and rounded leading edges, see Luckring (2013). In this case, the origin of the longitudinal vortices is displaced downstream along the leading edge. For the $R_{10} S_{10}$ configuration, the streamwise vorticity related to the vortex is less concentrated and weaker when compared with the $R_{10} S_{0}$ configuration (Figs. 4,5 ). The associated low-pressure footprint is also weaker and more localized at the bottom of the C-pillar. The pressure recovery on the slanted surface appears much more effective for $R_{10} S_{10}$ than for the other configurations (Figs. 3, 4, 5; Table 2). This point can justify the $18 \%$ lift reduction reported in Table 1 . Despite the reduction in the C-pillar vortex and the better pressure recovery on the slanted surface, no drag reduction was observed for the $R_{10} S_{10}$ case when compared with the $R_{10} S_{0}$ configuration (Table 1 ). The $C_{p}$ distribution on the vertical base (Fig. 5) and the corresponding average surface value (Table 2) indicate a clear decrease in pressure on the base $\left(-32 \%\right.$ compared with $\left.R_{10} S_{0}\right)$. $L^{*}=0.9$ for this configuration. In terms of drag generation, the unfavorable effect on the base balances exactly the positive effect on the slanted surface. Note that this zero net balance is of course specific to the shape of the rear end and, in particular, to the ratio between the base height and the vertical projection of the rear window, Howell and Good (2008).

Figure 6 shows the evolution of $\mathrm{Cp}$ along the symmetry plane for the three models more quantitatively. At the 

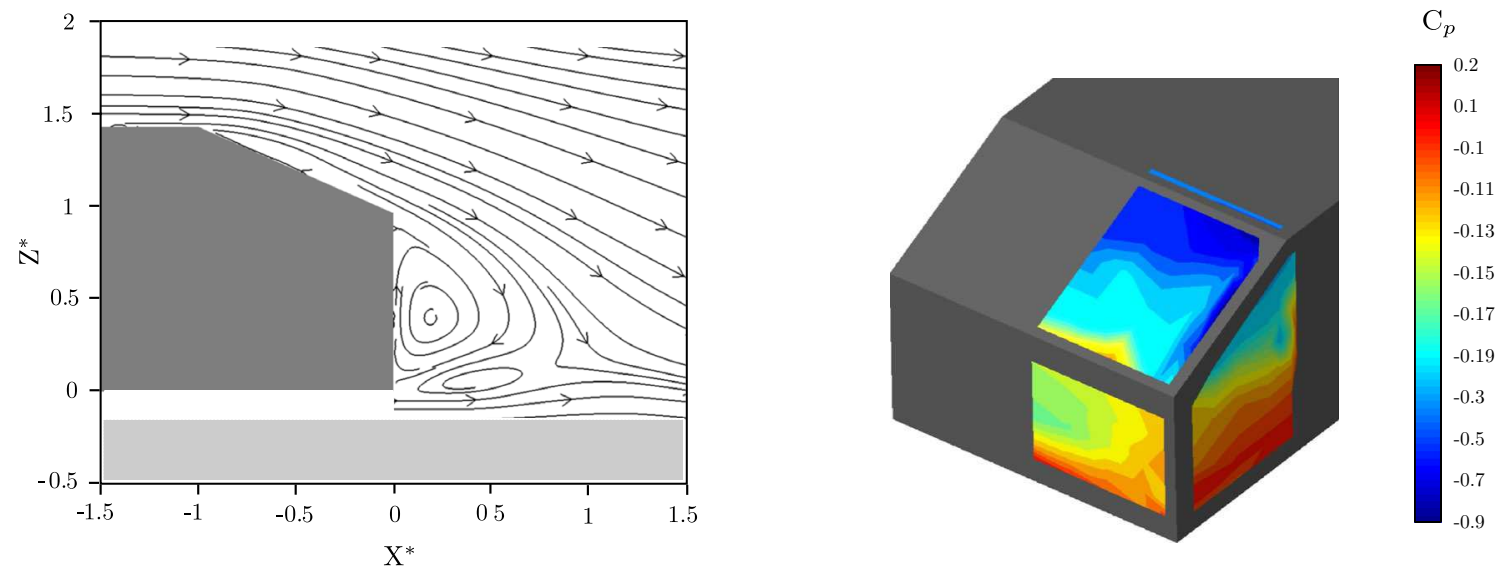

Fig. $3 R_{0} S_{0}$ Left time-averaged streamlines at $Y^{*}=0$. Right wall pressure coefficient $C_{p}$ on the right half
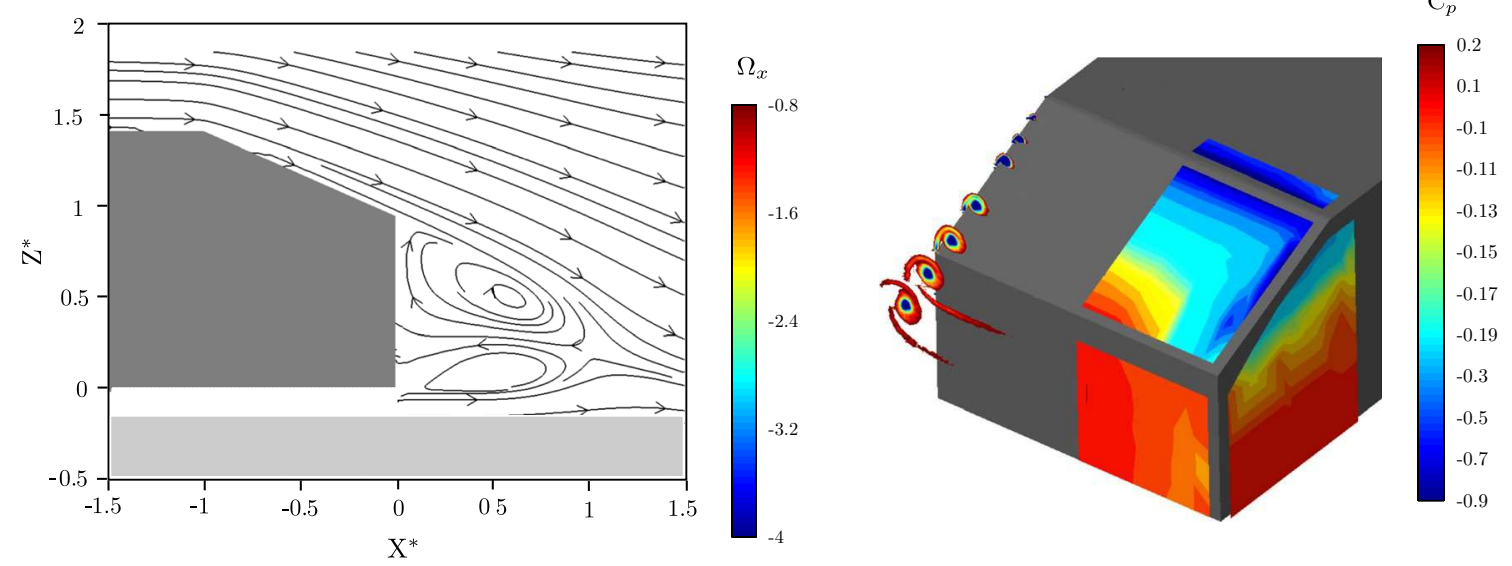

Fig. $4 R_{10} S_{0}$ Left time-averaged streamlines at $Y^{*}=0$. Right streamwise vorticity $\Omega_{x}$ on the left half and wall pressure coefficient $C_{p}$ on the right half
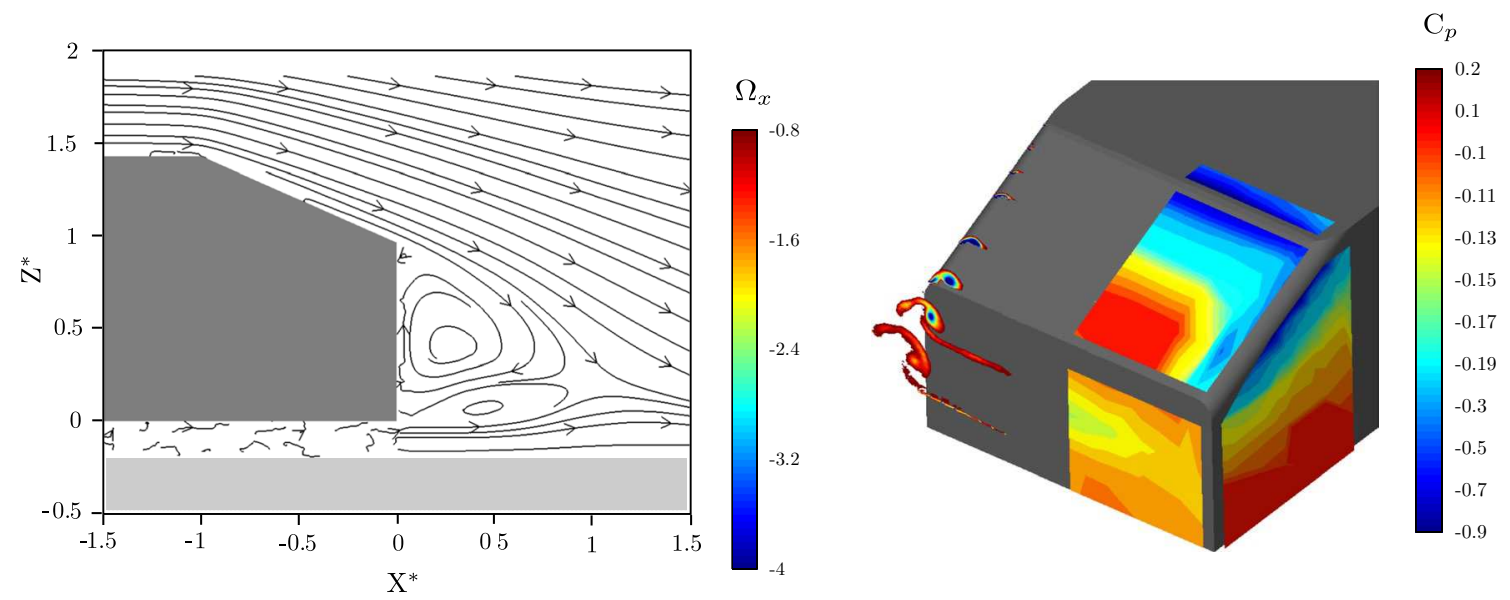

Fig. $5 R_{10} S_{10}$ Left time-averaged streamlines at $Y^{*}=0$. Right streamwise vorticity $\Omega_{x}$ on the left half and wall pressure coefficient $C_{p}$ on the right half 
Table 2 Variation in spatially averaged mean Cp over the rear window and vertical surface relative to the reference case $R_{0} S_{0}$

\begin{tabular}{lll}
\hline & Rear window $\Delta \mathrm{Cp}$ & Vertical surface $\Delta \mathrm{Cp}$ \\
\hline$R_{0} S_{0}$ & - & - \\
$R_{10} S_{0}$ & $+10 \%$ & $+28 \%$ \\
$R_{10} S_{10}$ & $+31 \%$ & $+5 \%$ \\
\hline
\end{tabular}

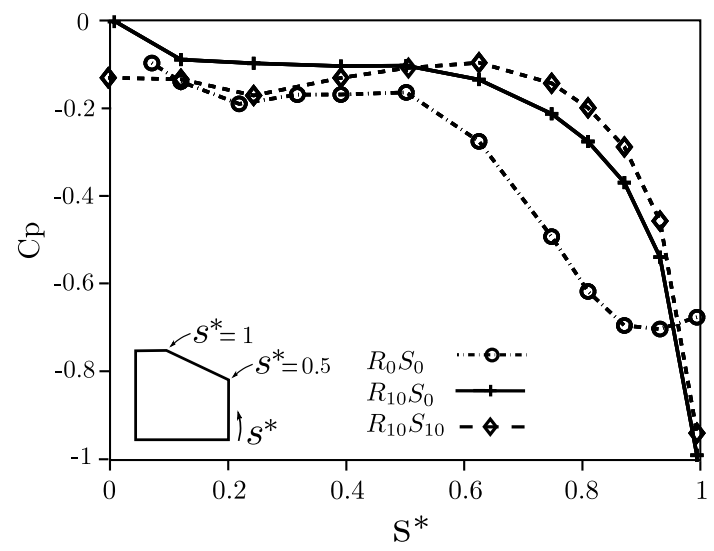

Fig. 6 Pressure distribution in the symmetry plane $\left(Y^{*}=0\right)$ for all models

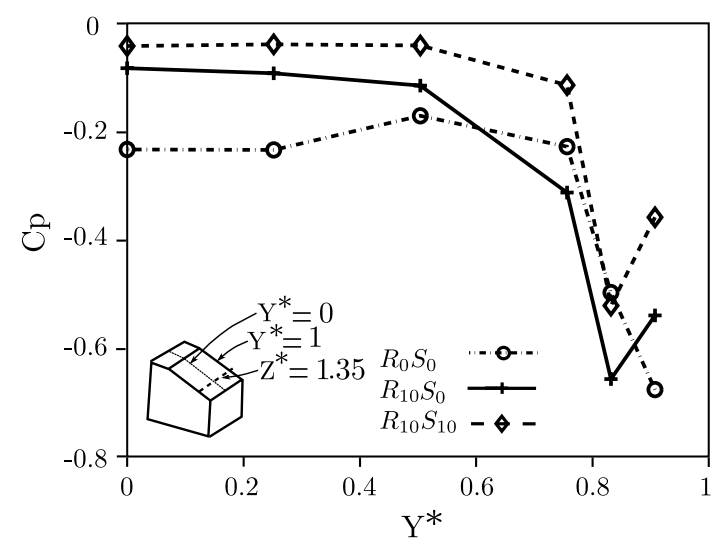

Fig. 7 Pressure distribution at constant $Z^{*}=1.35$ for all models

first probe along the slanted surface, $s^{*}=1$, the low $\mathrm{Cp}$ for $R_{10} S_{0}$ and $R_{10} S_{10}$ is due to the acceleration over the rounded edge on the roof. The sharp edge at the end of the roof generates a separation region, constant $\mathrm{Cp}$ up to $s^{*}=0.85$, for the reference case. On the contrary, the pressure coefficient of the models with a rounded edge on the top exhibits a pressure recovery all over the rear window. The pressure recovery is even larger for the $R_{10} S_{10}$ model compared with $R_{10} S_{0}$. However, the decrease in Cp along the vertical surface is noticeable for the $R_{10} S_{10}$, showing that the flow dynamics in the 3D wake downstream of the
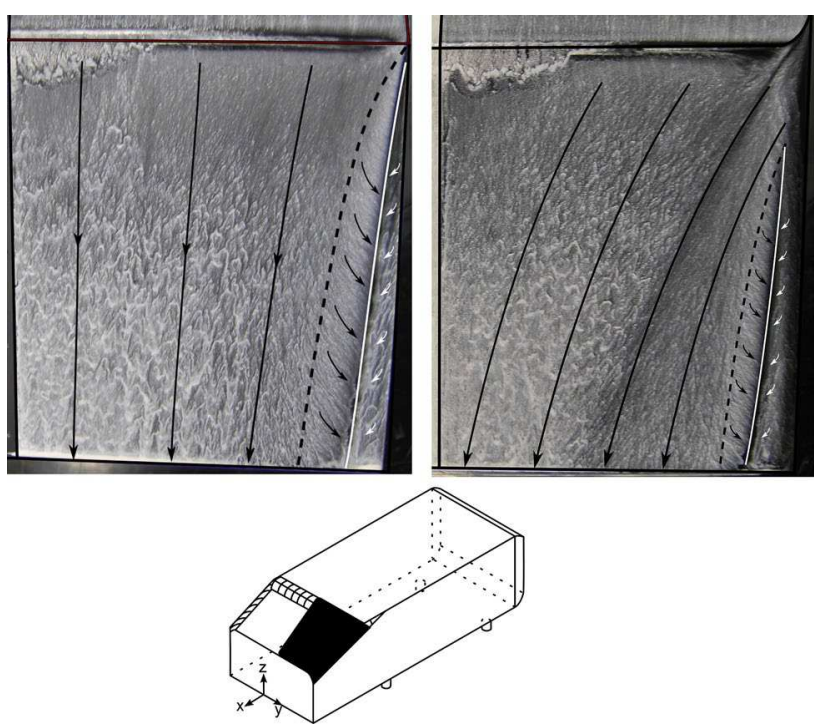

Fig. 8 Oil flow visualization: Left $R_{10} S_{0}$. Right $R_{10} S_{10}$. Dashed black line is the attachment line of the primary vortex, and solid white line is the separation line of the secondary vortex. Bottom Position of the paint visualization

rounded configuration results in a low-pressure footprint associated to drag penalty, as discussed previously.

Figure 7 reports the spanwise evolution of $\mathrm{Cp}$ for $Z^{*}=1.35$, (this location corresponds to $s^{*}=0.6$ in Fig. 6). Model $R_{10} S_{10}$ has clearly a higher $\mathrm{Cp}$ value, more uniform along the transverse direction with respect to $R_{10} S_{0}$. As stated by Fuller and Passmore (2014), the higher central downwash speed induced by the stronger trailing vortices for the $R_{10} S_{0}$ case is expected to contribute to the lower $\mathrm{Cp}$ values in comparison with $R_{10} S_{10}$ case. A weaker C-pillar vortex for $R_{10} S_{10}$ should also be responsible for the more uniform $\mathrm{Cp}$ distribution across the backlight observed in Fig. 7. The low intensity of the C-pillar vortex pressure footprint for $R_{10} S_{10}$ is also observed at $Y^{*}=0.9$.

\section{On the effect of pillar rounding on the near wake development}

Oil flow visualizations were performed to investigate the skin friction lines for both the $R_{10} S_{0}$ and $R_{10} S_{10}$ models, white and black lines were superimposed to the pictures to emphasize the main structures in Fig. 8. On the side of the model, the dashed black line represents the attachment line of the primary vortex and the solid white line the separation line of the secondary vortex [see Krajnović and Davidson (2005) for a complete description of the topology of the pillar vortex]. Closer to the center region, skin friction lines cannot be interpreted directly and long black arrows 
indicate the overall direction of the flow off the surface. The footprint of the C-pillar vortex is wider for the $R_{10} S_{0}$ case, and moreover, it is observed that the origin of the vortex is shifted downstream for $R_{10} S_{10}$. On the upper part of

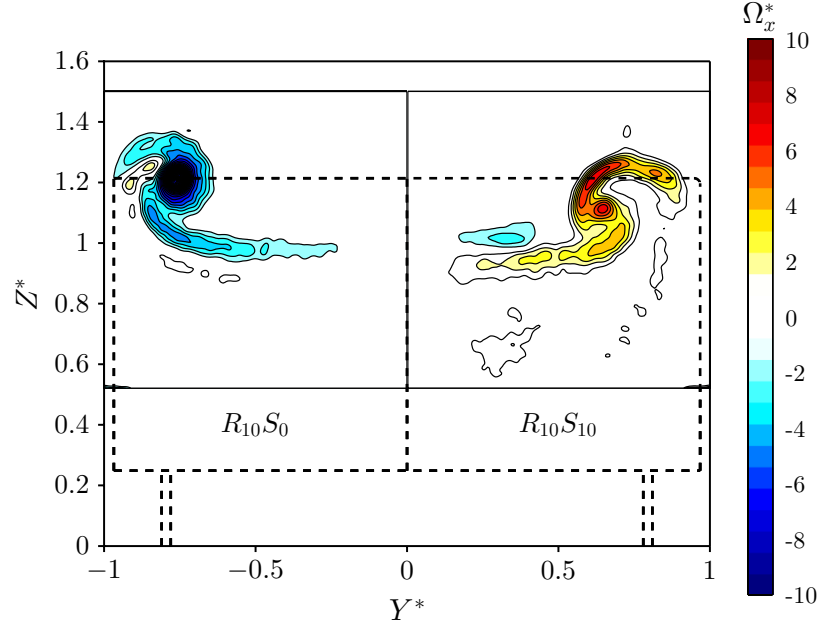

Fig. 9 Mean streamwise vorticity $\Omega_{x}^{*}=\frac{\Omega_{x} * \xi}{U_{0}}$, plane at $X^{*}=L^{*} / 2$. The dashed lines represent the geometry of the model
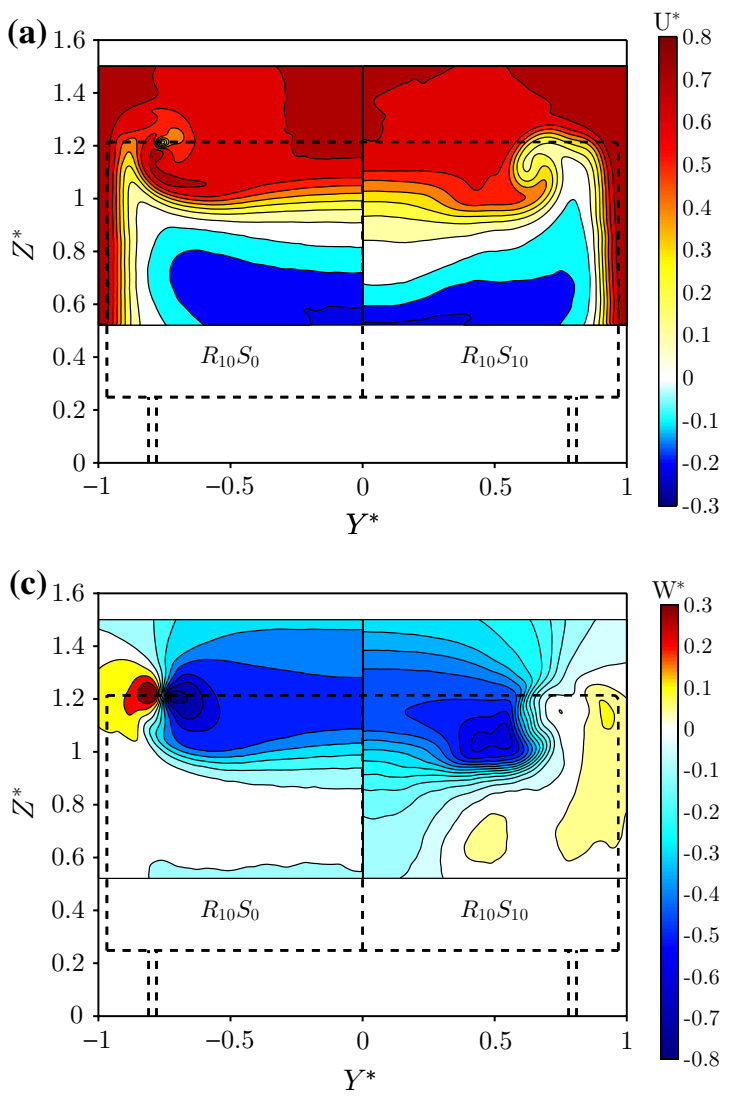

the rounded pillar, $R_{10} S_{10}$, some attached flow penetrates toward the center line. That flow comes from the side of the model and does not roll up directly in the C-pillar vortex as in the sharp case, but penetrates directly over the rear window. The analysis of the oil flow visualization brings to evidence the different topology of the skin friction lines over the rear window, due to the geometrical differences in the side rear pillars of the models.

Contours of the mean streamwise vorticity in the plane $X^{*}=L^{*} / 2$ are proposed in Fig 9. Small values of streamwise vorticity are not displayed, so as to focus the attention on the vortical structures generated from the side pillars. A concentrated region of vorticity is observed for $R_{10} S_{0}$ and corresponds to the longitudinal vortex. For the rounded case, the streamwise mean vorticity is distributed over a wider area of the near wake. A small region of negative vorticity is also observed for $\mathrm{Y}^{*} \approx 0.4$ and $\mathrm{Z}^{*} \approx 1$. A closer look at the oil visualization in Fig. 8 and to $V^{*}$ in Fig. $10 \mathrm{~b}$ shows that this occurs in the zone of confluence between the flow over the top of the body and the lateral flow drawn from the side. In that region, a clear change in sign of the gradient $\frac{\partial V^{*}}{\partial z}$ is observed, and this contributes to the change in sign of the streamwise vorticity.
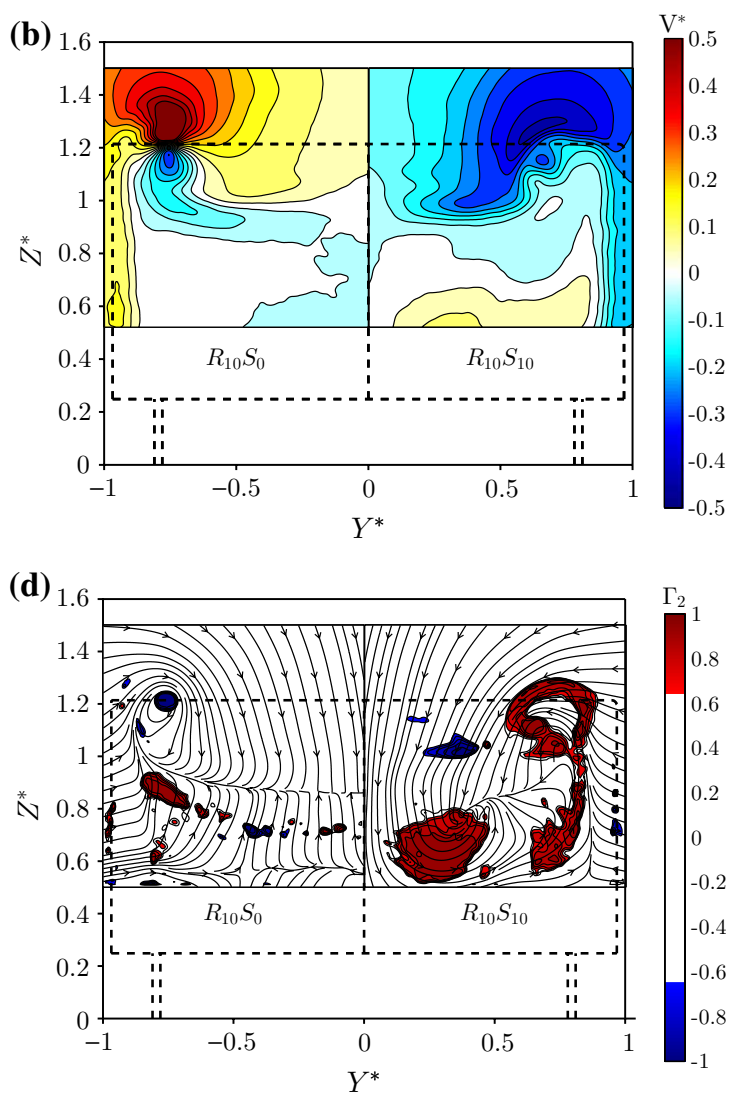

Fig. 10 Plane at $X^{*}=L^{*} / 2$. The dashed lines represent the geometry of the model, (a) mean streamwise velocity $U^{*}$, (b) mean spanwise velocity $V^{*},(\mathbf{c})$ mean normal velocity $W^{*},(\mathbf{d}) \Gamma_{2}$ 
Figure 10 shows the three components of the mean velocity field at $X^{*}=L^{*} / 2$. Each picture is divided into two: the left side represents $R_{10} S_{0}$ and the right side $R_{10} S_{10}$. The data are all recorded for $Y^{*} \in[0 ; 1]$, so the data visualization related to $R_{10} S_{0}$ is a mirror symmetry.

The different organization of the near wake flow should be noticed. In particular, the horizontal shear layer downstream separation is transported downward when rounded edges are present. The topology of the mean near wake flow and of the recirculation bubble is thus changed. When comparing the transverse velocities $\left(V^{*}\right)$ in the horizontal shear layer region downstream of the slanted surface, the inward motion is clear for $R_{10} S_{10}$ while significant inward $V^{*}$ values are just present around the vortex core for $R_{10} S_{0}$. For the rounded case, Fig. 10c shows that this mean inward motion before separation results in a strong downwash effect (negative $W^{*}$ ) in the core region of the wake.

For rear end with a slanted angle, a pressure gradient is induced between the side of the model and the rear end, Ahmed et al. (1984). As a consequence, the flow along the side is drawn in toward the center. The sharp side pillar forces the lateral flow to separate, generating a longitudinal vortex. One could therefore suggest that, for $R_{10} S_{0}$, the sharp lateral edges "shield" the wake separation region at the base and enable a "two-dimensional" separation, "2D" meaning that the mean streamlines at separation are more parallel to the orientation of the flow over the window, while longitudinal vorticity is concentrated in the C-pillar vortices. On the contrary, for rounded edges, the flow is drawn in from the sides, toward the center over the slanted surface. The separation at the base is then notably 3D for $R_{10} S_{10}$, associated with high spanwise and vertical velocity components.

The contours of $\Gamma_{2}$, computed using $V^{*}$ and $W^{*}$ velocity components, report the rotational behavior of the flow. Two-dimensional lines tangent at each point to the vector $\left(V^{*}, W^{*}\right)$ are also drawn in Fig 10d. Of course, these lines are not meant to be mean streamlines. They are drawn here because they clearly show the overall rotation of the $R_{10} S_{10}$ wake around the longitudinal axis. For $R_{10} S_{0}$, a concentrated region of $\Gamma_{2}$ corresponds to the C-pillar vortex. For $R_{10} S_{10}$, the criterion reveals a zone of coherent rotation around the pillar vortex and a large zone in the center bottom region of the wake. The high spanwise and vertical velocity associated with the transversal flow therefore results in a global rotation of the flow in the near wake that will be discussed more quantitatively in what follows (see Fig. 12 and associated comments). The downwash effect is also particularly clear when looking at Fig. 11, which compares the turbulent kinetic energy at $X^{*}=L^{*} / 2$. The 3D shear layers and the wake recirculating region (see the region of negative $U^{*}$ in Fig. 10a)

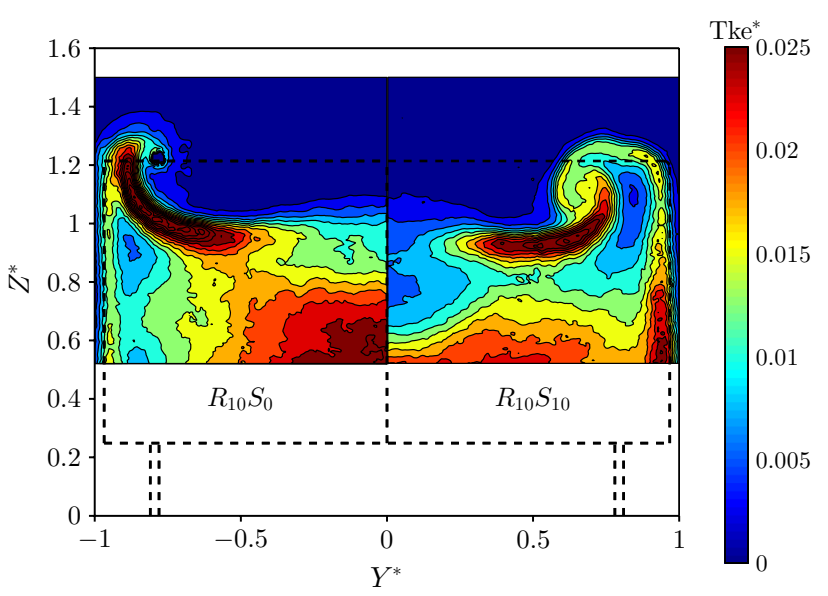

Fig. 11 Turbulent kinetic energy Tke*, plane at $X^{*}=L^{*} / 2$. The dashed lines represent the geometry of the model

are the location of a large fluctuating level. For $R_{10} S_{10}$, the "potential" outer flow, characterized by a low level of turbulent kinetic energy, penetrates downward in the near wake. As a consequence, the fluctuating kinetic energy is much lower in this region, as shown in the zone around $Y^{*} \in[0 ; 0.2]$ and $Z^{*} \in[0.6 ; 1]$. For rounded rear ends, the three-dimensional property of the near wake structure therefore results in more pronounced streamline curvatures and lower pressure on the base when compared with a similar model featuring sharp lateral edges. Note that the downward deviation of the mean streamlines separating from the slanted surface is particularly manifest when looking at the orientation of the streamlines in the plane of symmetry in Fig. 5.

Along three-dimensional geometries, 3D vorticity is generated at the wall along the curved surface, transported, tilted, and stretched in the 3D boundary layer region. In what follows, it is proposed to discuss some integral properties of the longitudinal vorticity field along the near wake of the body. One specificity and limitation of the data presented is that, contrary to the recent study of Venning et al. (2015), the detailed stereo-PIV database only concerns velocity planes measured near the rear vertical surface. This region spans approximately over half of the mean recirculating bubble (see Figs. 4, 5) where the mean recirculating flux is significant. Moreover, the model used here being four times as large as the body of Venning et al., the field of view of each plane does not include the full height of the wake. In particular, the under-hood flow and the bottom region of the wake are not captured. The following discussion must therefore be considered as qualitative. It was however checked that all conclusions discussed here are consistent with the results from all numerical simulations performed by Rossitto et al. (2014). 


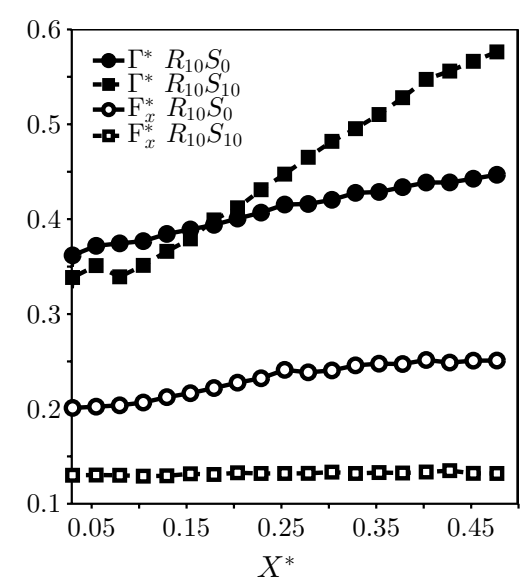

Fig. 12 Longitudinal evolution of the mean circulation and the flux of mean longitudinal vorticity in the near wake

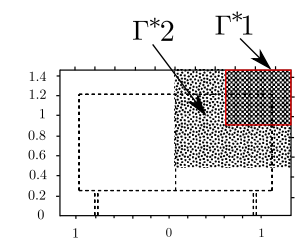

\begin{tabular}{c|c|c|} 
& $R_{10} S_{0}$ & $R_{10} S_{10}$ \\
\hline$\Gamma^{*} 1$ & 0.42 & 0.30 \\
\hline$\Gamma^{*} 2$ & 0.02 & 0.26 \\
\hline$\Gamma^{*}=\Gamma^{*} 1+\Gamma^{*} 2$ & 0.44 & 0.56 \\
\hline
\end{tabular}

Fig. 13 Zone of calculation and values of circulation at $X^{*}=L^{*} / 2$. The dashed lines represent the geometry of the model

The longitudinal evolution of the mean circulation $\Gamma^{*}=\int_{S^{*}} \Omega_{x}^{*} d S$ and the flux of mean longitudinal vorticity $F_{x}^{*}=\int_{S^{*}} U^{*} \Omega_{x}^{*} d S$ are plotted in Fig. 12 for both configurations. As circulation is the integral of the longitudinal vorticity, it is natural to consider the balance of $\Omega_{x}$ as a constitutive element of the structuration of the wake.

First, it is noticed that $F_{x}^{*}$ is in the order of 0.13 for $R_{10} S_{10}$ and 0.25 for $R_{10} S_{0}$ at $X^{*}=L^{*} / 2 . F_{x}^{*}$ is a nondimensional quantity, in which the scaling quantity is $\xi U_{0}^{2}$. The scaling length $\xi$, defined in Fig. 2, is equal to $201 \mathrm{~mm}$. The length $l$ of the perimeter of the vertical base of the model is about $l \approx 5.8 \xi$. In the ideal case of a 2D boundary layer of thickness $\delta$ and external velocity $U_{0}$ separating at a sharp edge, the absolute value of the flux of vorticity per unit span reads simply as $\left|F_{\perp}\right|=\int_{0}^{\delta} U \frac{\partial U}{\partial n} d n=\frac{U_{0}^{2}}{2}$ where $\mathrm{n}$ is the direction perpendicular to the wall and $-\frac{\partial U}{\partial n}=\Omega_{\perp}$ denotes the only nonzero component of the vorticity vector. By integration of the absolute value of the vorticity flux along the perimeter of the vertical base, an order of magnitude of the total longitudinal mean flux of vertical and transversal mean vorticity shed at the base of the body is therefore $F_{Y Z} \approx \frac{U_{0}^{2} l}{2}$. Comparing this order of magnitude with $2 F_{X}$ ( $F_{X}$ concerns approximately half of the wake surface), we obtain $2 F_{X} / F_{Y Z} \approx 4 \xi F_{X}^{*} / l \approx 2 F_{X}^{*} / 3$ that is to say $2 F_{X} / F_{Y Z} \approx 0.08$ for $R_{10} S_{10}$ and $2 F_{X} / F_{Y Z} \approx 0.16$ for $R_{10} S_{0}$. It is therefore shown that the flux of organized longitudinal vorticity is only a small portion of the base flux. However, this experiment shows that it has a significant global incidence on the pressure distribution and drag of the body.

The second observation is that $F_{X}^{*}$ is quasi-constant for $R_{10} S_{10}$ and increases moderately for $R_{10} S_{0}$ (a $25 \%$ increase over the measurement region). Integrating the longitudinal vorticity equation in a control volume bounded by two measurement planes at $X^{*}$ and $X^{*}+\Delta X^{*}$, the longitudinal evolution of $F_{X}^{*}$ is due to the flux of $\Omega_{x}^{*}$ across the lateral boundaries and to the integral of the source terms in the control volume (viscous diffusion can be neglected at this Reynolds number; turbulent transport was not evaluated but should be zero along the plane of symmetry and negligible if the boundaries of the control volume are out of the wake, which is not the case here for the base). The source terms correspond to the average of the stretching of longitudinal vorticity $\Omega_{x} \frac{\partial U}{\partial x}$ and of the tilting of lateral and vertical vorticity $\Omega_{y} \frac{\partial U}{\partial y}+\Omega_{z} \frac{\partial U}{\partial z}$ (Tennekes and Lumley 1972; Chassaing 1990). In the present case, it was checked that the non-dimensional lateral and vertical fluxes are significantly smaller than the longitudinal flux of vorticity (smaller than $5 \%$ of $F_{X}^{*}$ ). Figure 12 shows that the net effect of the source terms over the measurement volume is small for $R_{10} S_{0}$ and negligible for $R_{10} S_{10}$. From an integral point of view, the longitudinal mean transport of mean longitudinal vorticity seems therefore to be the dominant term in the near wake.

The third observation relative to Fig. 12 is that, despite the constancy of the flux, the integral of the vorticity over the measurement domain, circulation $\Gamma^{*}$, increases significantly. The rate of increase is notably greater for $R_{10} S_{10}$ than for $R_{10} S_{0}$, about three times as much for the former as for the latter. To understand simply this observation, it was chosen to divide the total domain in two fixed parts (see sketch in Fig. 13). This partition is quite arbitrary, but is sufficient for the discussion. Region 1 includes the C-pillar vortex and covers $1 / 4$ of the field of measurements: $Y^{*} \in[0.44,0.89], Z^{*} \in[1,1.5]$. Region " 2 " is the remaining area. $\Gamma^{*} 1$ (respectively $\left.\Gamma^{*} 2\right)$ represents the circulation associated with region 1 (respectively 2). Naturally, we have $\Gamma^{*}=\Gamma^{*} 1+\Gamma^{*} 2$. Figure 13 displays this decomposition at $X^{*}=L^{*} / 2$. It is clear that circulation $\Gamma^{*} 1$ in the region including the vortex is larger for $R_{10} S_{0}$ than for $R_{10} S_{10}$. This is related to the higher circulation gathered in the C-pillar vortex. However, while $\Gamma^{*} 2$ is less than $5 \%$ of the total value of $\Gamma^{*}$ for $R_{10} S_{0}$, it is about $50 \%$ of the total circulation for $R_{10} S_{10}$. The $3 \mathrm{D}$ wake generated by rounded C-pillar therefore significantly differs from the sharp pillar configuration in 
that the longitudinal vorticity is transported in the core of the wake in a more distributed way. This is consistent with Fig. 9 in which this observation was already made. The fact that the flux $F_{X}^{*}$ is a constant is surprising but does not contradict this observation, because a significant part of the vorticity distributed in region 2 is associated with a reversal flow. For the rounded case, this upstream transport of longitudinal vorticity very close to the base of the body is responsible for low-pressure regions and more drag. It will be shown in the "Appendix" that this effect is amplified if rounding of the back pillar is more pronounced.

\section{Conclusions}

Analysis has been performed to gain understanding in the effect of afterbody rounding on the generation of aerodynamic forces on a blunt body. Simplified vehicle models with a 25 degrees rear slant presenting different upper and side edge curvatures have been investigated. Similarly to previous studies, rounding the upper edge was shown to reduce drag up to $16 \%$, linked to the separation suppression on the slanted surface and the lengthening of the separation region close to the vertical base. For this particular geometry, surprisingly, additional rear curvature associated with pillar rounding did not further improve the drag. However, the zero net effect was found to result from opposite behaviors of the flow on the slanted and vertical surfaces and to hide strong local modifications of the flow field. When rounding the side edge, the C-pillar vortex becomes weaker and a better pressure recovery is observed on the slanted surface. Both of these effects are favorable to the reduction in lift and drag. Furthermore, for this rounded configuration, the lateral flow not only feeds the C-pillar vortex but part of it penetrates the slanted surface toward the center line. This mean inward motion before separation has been found to promote a strong downwash in the core region of the wake. The resulting three-dimensional near wake exhibits a more pronounced streamline curvature and a lower pressure on the base when compared with a similar model with sharp lateral edges. It is suggested that the upstream transport of mean streamwise vorticity next to the vertical base is responsible for low-pressure regions. In terms of drag, this unfavorable pressure distribution on the base counterbalances the gain obtained in the slanted surface for this particular geometry. Additional experimental tests, reported in the "Appendix" were performed to understand the effect of rounding the side pillars of a realistic model. Similarly to the Ahmed model, the flow field past the rear end is strongly modified despite a small variation in drag.
Acknowledgments The authors are thankful to L. Mies who took care of the production process of the models and to Y. Goraguer, the wind tunnel manager, for his precious help during the experiments. We acknowledge A. Glad for reviewing the English of the paper. This work has been performed in the framework of the "OpenLab Fluidics"@Poitiers.

\section{Appendix}

The present work pointed out that a small edge radius on the side of a simplified car model modifies the flux and the orientation of the mean vorticity vector. One should not forget that realistic cars tend to have large radii of curvature. This justifies the need to test the effect of a large radius of curvature over a realistic car shape to prove that the $R_{10} S_{10}$ rear end of the Ahmed model is an intermediate configuration where C-pillar vortices are delayed but still present in a weaker way. The aim of this complementary test was to increase the physical understanding of the phenomena and prove that what was understood thanks to the Ahmed body rounding can be exploited for more realistic models.

The chosen model and its relevant dimensions are exhibited in Fig. 14. Two different rear ends were tested: one with a sharp lateral radius and one with an edge radius corresponding to $40 \%$ of the span of the vehicle. Both rear ends have a rounded edge at the end of the roof to avoid flow separation over the rear window.

Table 3 reports the variation in the drag and lift coefficients for the rounded rear end compared with the sharp one, (the value of drag and lift is not reported because they are meaningless since this model is a unique prototype). As an order of magnitude, the drag coefficient

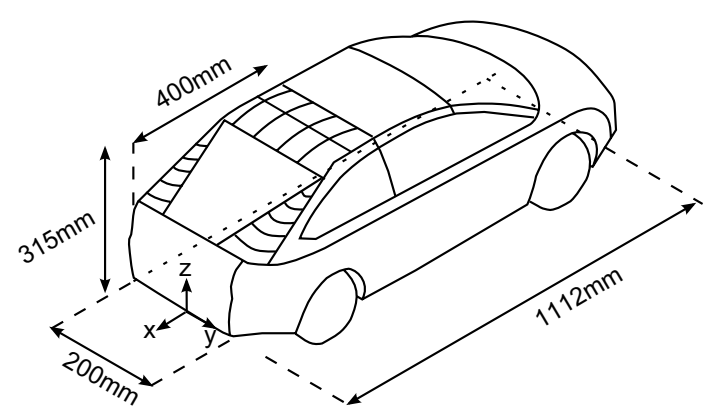

Fig. 14 Realistic model and location of the rounded edges

Table 3 Variation in aerodynamic coefficients relative to the sharp case

\begin{tabular}{lll}
\hline & $\Delta \mathrm{Cd}$ & $\Delta \mathrm{Cl}$ \\
\hline Sharp & - & - \\
Rounded & $+1.5 \%$ & $-8.5 \%$ \\
\hline
\end{tabular}


of the sharp rear end is $35 \%$ less than the reference Ahmed body. The rounded configuration exhibits a slight increase in drag. As for the Ahmed body, this hides major local flow modifications. On the contrary, lift is strongly reduced, which demonstrates the influence of the rounded edge on the C-pillar.

When comparing with Fig. 8, similar oil flow visualization interpretations were made for the realistic model in Fig. 15. The sharp configuration, reported on the left side of the picture, clearly demonstrates the presence of a C-pillar vortex. For the rounded configuration, the large curvature does not even generate a delayed C-pillar vortex; all the flow that comes from the side of the model, in fact, enters the rear window region through an inward transverse attached flow.

Figure 16 highlights the pressure wall measurements over the rear ends. Due to the complexity of the side surface of the model, as for example a realistic gap between the window and the side surface of the model, the C-pillar vortex pressure footprint is less intense than the modified Ahmed body, but still present in the sharp case. The pressure distributions on the side of the rounded edge rear end confirm the absence of the C-pillar vortex. As with the modified Ahmed body, the

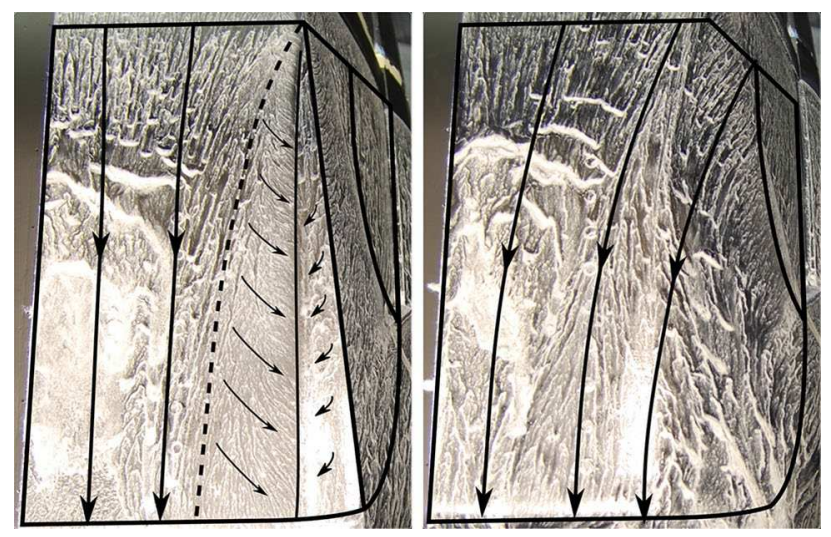

Fig. 15 Oil visualization. Left sharp rear end. Right rounded rear end. Dashed line is the attachment line of the primary vortex, and solid line is the separation line of the secondary vortex. Long arrows indicate the overall direction of the flow off the surface
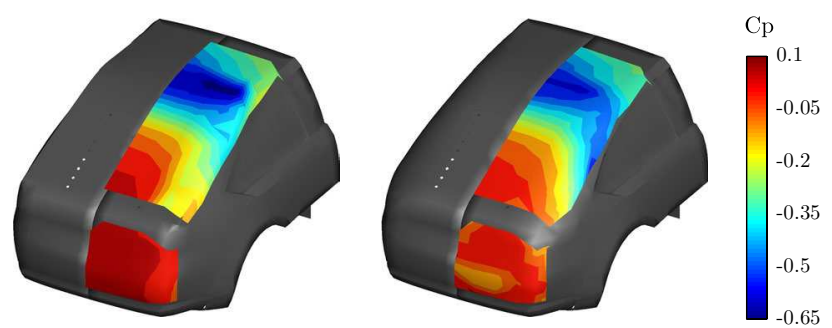

Fig. 16 Wall pressure coefficient $C_{p}$ : left sharp, right rounded
Table 4 Variation in spatially averaged mean $\mathrm{Cp}$ over the rear window and vertical surface relative to the sharp case

\begin{tabular}{lll}
\hline & Rear window $\Delta \mathrm{Cp}$ & Vertical surface $\Delta \mathrm{Cp}$ \\
\hline Sharp & - & - \\
Rounded & $+10 \%$ & $-300 \%$ \\
\hline
\end{tabular}

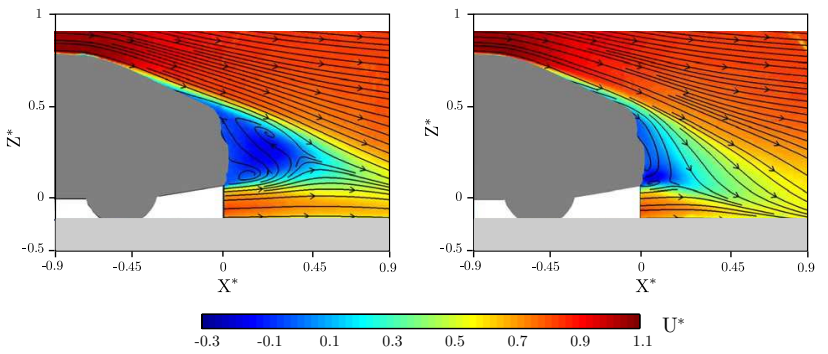

Fig. 17 Streamwise velocity $U^{*}$ at $Y^{*}=0$. Left sharp rear end. Right rounded rear end

vertical surface displays a lower pressure coefficient than for the sharp case, Table 4. For the Ahmed body, the loss of pressure on the vertical surface compensates for the gain over the rear window when a rounded edge is present. This is not the case for the realistic model, where the net balance between the loss and the gain is not zero $(\Delta \mathrm{Cd}=+1.5 \%$, see Table 3 ). This underlines the generic nature of the positive and negative effects over the two zones of the rear end, but points out that every model has its own ratio between the vertical surface and the rear window, which influences drag balance, Howell and Good (2008).

PIV was also performed in the symmetry plane of the model Fig. 17. It is noticed that the rounding of the C-pillar induces strong effects in the near wake close to the vertical surface. When rounding the C-pillar, the length of the separated region is strongly reduced and no "spanwise" largescale rotation is detected in the symmetry plane. In fact, the separated zone is now a fully 3D flow. To understand this modification of topology in the near wake, YZ stereo-PIV planes are reported in Fig. 18 at $X^{*}=0.07$.

Figure 18a evidences the presence of the C-pillar vortex in the sharp case due its characteristic streamwise velocity contours, no sign of the vortex is visible on the right side of the picture. On the top of the separated region, the vortex generated from the front of the vehicles, the so-called A-pillars, is visible for both models. The spanwise velocity is reported in Fig. 18b. While for the sharp rear end there are two opposite regions of localized transverse velocity due to the C-pillar vortex, for the rounded rear only an intense region of lateral flow that comes from the side (and is injected in the near wake) is present. The resulting downwash is visible in the right 

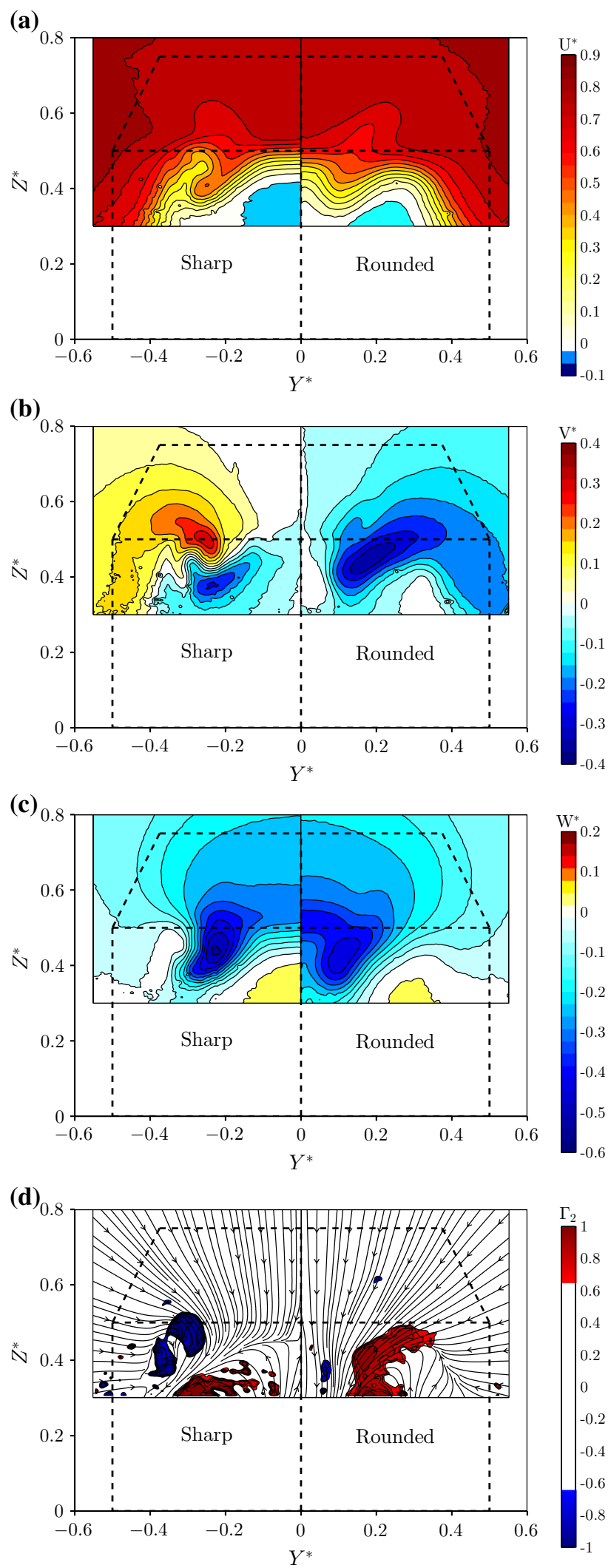

Fig. 18 Plane at $X^{*}=0.07$. The dashed lines represent the geometry of the model, (a) mean streamwise velocity $U^{*},(\mathbf{b})$ mean spanwise velocity $V^{*}$, (c) mean normal velocity $W^{*}$, (d) $\Gamma_{2}$ side of Fig. 18c. Contours of $\Gamma_{2}$ in Fig. 18d illustrate the global streamwise rotation of the flow, expected to induce a low-pressure region over the vertical base of the vehicle.

\section{References}

Ahmed S, Ramm G, Faitin G (1984) Some salient features of the time-averaged ground vehicle wake. Technical report, Society of Automotive Engineers Inc, Warrendale, PA

Beaudoin J, Aider J (2008) Drag and lift reduction of a 3D bluff body using flaps. Exp Fluids 44(4):491-501

Chassaing P (1990) Turbulence en mécanique des fluides: analyse du phénomène dans une perspective de sa modélisation à l'usage de l'ingénieur

Conan B, Anthoine J, Planquart P (2011) Experimental aerodynamic study of a car-type bluff body. Exp Fluids 50(5):1273-1284

Davis JP (1982) Wind tunnel investigations of road vehicle wakes. $\mathrm{Ph} . \mathrm{D}$ thesis, Imperial College London (University of London)

Fuller J, Passmore MA (2014) The importance of rear pillar geometry on fastback wake structures. J Wind Eng Ind Aerodyn 125:111-120

Graftieaux L, Michard M, Grosjean N (2001) Combining PIV, POD and vortex identification algorithms for the study of unsteady turbulent swirling flows. Meas Sci Technol 12(9):1422

Howell J, Good GL (2008) The effect of backlight aspect ratio on vortex and base drag for a simple car-like shape. SAE

Howell J, Hickman D (1997) The influence of ground simulation on the aerodynamics of a simple car model. SAE

Joseph P (2012) Application du contrôle par jets pulsés à un corps non profilé. Ph.D thesis, Université Pierre et Marie Curie-Paris VI

Joseph P, Amandolèse X, Aider JL (2012) Drag reduction on the 25 slant angle ahmed reference body using pulsed jets. Exp Fluids 52(5):1169-1185

Katz J (1995) Race car aerodynamics: designing for speed (engineering and performance). Bentley Publishers, Cambridge

Krajnović S, Davidson L (2005) Flow around a simplified car, part 2: understanding the flow. J Fluids Eng 127(5):919-928

Luckring JM (2013) Initial experiments and analysis of blunt-edge vortex flows for VFE-2 configurations at NASA Langley, USA. Aerosp Sci Technol 24(1):10-21

Rossitto G, Sicot C, Ferrand V, Borée J, Harambat F (2014) Influence of the afterbody rounding on the pressure forces of the Ahmed body. In: First international conference in numerical and experimental aerodynamics of road vehicles and trains. Aerovehicles1

Rossitto G, Sicot C, Ferrand V, Borée J, Harambat F (2015) Wake structure and drag of vehicles with rounded rear edges. In: 50th international conference on applied aerodynamics. 3AF

Tennekes H, Lumley JL (1972) A first course in turbulence. MIT Press, Cambridge

Thacker A, Aubrun S, Leroy A, Devinant P (2012) Effects of suppressing the $3 \mathrm{~d}$ separation on the rear slant on the flow structures around an ahmed body. J Wind Eng Ind Aerodyn 107:237-243

Venning J, Jacono DL, Burton D, Thompson M, Sheridan J (2015) The effect of aspect ratio on the wake of the ahmed body. Exp Fluids 56(6):1-11 